\title{
Assessing negative priming by attended distractors in a paper-and-pencil task
}

F.M. Rosin

\begin{abstract}
The paper-and-pencil digit-comparison task for assessing negative priming (NP) was introduced, using a referent-size-selection procedure that was demonstrated to enhance the effect. NP is indicated by slower responses to recently ignored items, and proposed within the clinical-experimental framework as a major cognitive index of active suppression of distracting information, critical to executive functioning. The digit-comparison task requires circling digits of a list with digit-asterisk pairs (a baseline measure for digit-selection), and the larger of two digits in each pair of the unrelated (with different digits in successive digit-pairs) and related lists (in which the smaller digit subsequently became a target). A total of 56 students (18-38 years) participated in two experiments that explored practice effects across lists and demonstrated reliable NP, i.e., slowing to complete the related list relative to the unrelated list, $(\mathrm{F}(2,44)=52.42, \mathrm{P}<0.0001)$. A 3rd experiment examined age-related effects. In the paper-and-pencil digit-comparison task, NP was reliable for the younger $(\mathrm{N}=8,18-24$ years) and middle-aged adults ( $\mathrm{N}=8,31-54$ years), but absent for the older group ( $\mathrm{N}=8,68-77$ years). NP was also reduced with aging in a computer-implemented digit-comparison task, and preserved in a task typically used to test location-specific NP, accounting for the dissociation between identity- and spatial-based suppression of distractors $(\operatorname{Rao} \mathrm{R}(3,12)=16.02, \mathrm{P}<0.0002)$. Since the paper-andpencil digit-comparison task can be administered easily, it can be useful for neuropsychologists seeking practical measures of NP that do not require cumbersome technical equipment.
\end{abstract}

\section{Introduction}

During the last few decades, there has been an increasing interest in the inhibitory control of the flow of visual processes into actions, which may be evoked even when there is little or no conscious intention to act. Measures of the capacity to resist a prepotent response of the moment are difficult to be estimated directly from performance in
Key words

- Negative priming

- Selective attention

- Paper-and-pencil tasks

- Aging

- Executive function

.......
Received February 19, 2003

Accepted April 7, 2004 normal human subjects, and therefore it has been necessary to develop indirect measures of performance such as priming procedures. An increasing attempt has been made to develop pencil-and-paper neuropsychological tests which do not require cumbersome technical equipment, in order to simplify their day-to-day clinical use and to permit standardization (1).

The objective of the present study was to 
introduce the digit-comparison task, a new paper-and-pencil task from which the negative priming (NP) effect has been reliably indexed in a rapid and easy way, and to document its suitability for detecting individual differences within the clinical-experimental neuropsychology setting.

There is evidence that NP may reflect a mechanism of control, crucial for coherent behavior, expressed when directing responses toward a target stimulus in the presence of a concurrent distractor which competes for the control of action. NP, also known as distractor suppression effect, has been typically observed in selective attention tasks that require selecting and responding to targets while disregarding distracting information (2). The statement that attention theorists have argued about is that there exists a limitation of processing capacity, so that selection of and responses to the relevant aspects of the environment may entail the active ignoring of that which does not serve the current goals. The implication is that this incredibly efficient processing of selective attention produces a normal slowing in time or is more error-prone in selecting an item, when it was selected against in the preceding trial. This effect was called NP, and it has been defined as a significant time cost in responding to a target that shared features with the distractor recently ignored in the previous trial, when compared with response times to a new target (3). For example, people usually take more time to report the identity or location of an item when in the preceding trial they were supposed to ignore an identical distractor or a distractor presented at the same site, respectively.

Slowing for recently ignored targets in priming tasks generally reaches modest magnitude, with values of less than $20 \mathrm{~ms}$ (4). Therefore, computerized procedures are usually implemented to measure NP more precisely. It was demonstrated that tasks requiring overt attention to distractors provided enhanced measures of NP (5). Thus, for the paper-and-pencil digit-comparison task, a referent size-selection procedure was chosen, which required attending to both target and distractors, i.e., the subject was asked to compare digit pairs and select the highest digit. Because of both simplicity in its material and procedure and the larger magnitude of NP that is obtained by means of its referent size-selection procedure, a task such as the paper-and-pencil digit-comparison task would be of interest to neuropsychologists seeking practical ways to measure NP, which has evolved into an important research area. NP has been regarded as the best available index of active suppression of information irrelevant to the current goals in selective attention tasks and has been proposed for the detection of syndromes that involve cognitive impairment (6).

NP has been broadly investigated because of its relevance to theories of selective attention and its promising clinical applicability, since various clinical and developmental populations with increased interference from intrusive irrelevant information often fail to present NP. Absent or diminished NP was reported from neuropsychological studies with people who are supposed to show impaired inhibitory control in selective attention, although not all were more susceptible to interference from a distractor, e.g., adults with schizophrenia (7), focal cerebral damage $(8,9)$, Alzheimer's disease (10), and Huntington's diseases (11). Developmental stage studies also demonstrated that young children and older adults often do not demonstrate reliable NP in selection tasks that require a response to the identity of a target $(6,12-15)$. The findings were interpreted as a deficiency in inhibitory attentional mechanisms and were taken as evidence consistent with the view that NP is a marker of the residual inhibition associated with the previous irrelevant stimuli (16). According to this theory, response times to the recently ignored targets should be delayed by suppressing their internal representations, or by block- 
ing from potential effectors, suggesting that distractors are processed beyond physical features.

Much of the literature is consistent with selective attention processes underlying NP, but evidence from several studies challenges this theory and alternative and/or complementary theoretical accounts for NP involving memory processing have been proposed $(13,17)$. Memory-based models explain that NP may be caused by a conflict in the retrieval episode, such that the current stimulus cues the retrieval of most recent processing instances involving that stimulus and conflicts with the current response, rather than being directly caused by a process of active suppression of the distractor in the preceding trial (18). The memory-based construct also should predict reduced NP for older adults, because they should be less likely to engage in retrieval of previous episodes of the stimuli.

In contrast with the hypothesis of a pure inhibition mechanism applied to distractors during the prime trial causing NP, an episodic retrieval mechanism and item information mismatching might predict a positive relation rather than an inverse relation between NP and interference. In fact, a number of studies have indeed found that conditions that induced a greater magnitude of interference also induced a greater magnitude of NP, even though these two distractor processing indices can be dissociated in the individual performance (8; for review, see Ref. 4). Thus, the efficiency in selecting (lesser interference) by increased inhibition of a distractor might be related to higher NP in the subsequent trial, supporting an inhibitory effect, whereas a positive relation between higher interference by more in-depth processing of the distractor and increased NP could be explained by other mechanisms accounting for NP. According to an episodic-retrieval theory, stronger distractors may be more likely to be encoded as "to be ignored", and consequently they would be less likely to be selected in the subsequent trial; hence inter- ference and NP might correlate positively. However, a positive relation between NP and interference does not exclude the inhibitory explanation. From the inhibitory perspective, inhibition is usually viewed as reactive to the source of interference; therefore, a positive correlation between NP and interference may be explained by a reactive inhibition mechanism implemented when distractor interference is high, so that distractors intrude more in the control of action. On the other hand, when interference is treated as an individualdifference variable, individuals who are more susceptible to interference are predicted to show diminished NP because they may be less able either to inhibit the distractor (such as the inhibitory account proposes) or to encode the distractor and retrieve the priming episode (according to the episodic-retrieval account). Furthermore, NP may involve the operation of both attention-based and memory-based mechanisms $(19,20)$ during selection of the target from the distractor at encoding and during memory retrieval at analysis of the subsequent selection, and a variety of conditions in the experimental design may result in NP tasks that favor one type of processing or the other.

Although the review of the empirical evidence broadens the scope of the present study, the relation between distractor interference and NP and its possible dissociation under some circumstances have been of special relevance for neuropsychological studies over the last decades. NP may constitute an executive measure that interfaces perception and action depending upon task demands and directs attention resources toward goal-relevant activity, which discriminates individuals who do not seem to differ in their pure measures of efficiency of selective attention. Thus, NP may be an expression of the executive role of attention in suppressing an unattended object to prevent response conflict and in providing coherence between the conscious experience and action streams (19). 
NP has been shown to be reliable under a very wide range of stimuli and procedures and different response modalities (21), and across practice (22). NP has been reported from a variety of computer-implemented tasks such as color naming tasks (23), identity-based tasks which require attending an item specified by a perceptual cue (e.g., symbol identity, color, location) while ignoring a distractor, location-based tasks which require selecting the target on the basis of a physical attribute but reporting the location of the target (24), same-different tasks that ask participants to match and judge items as the same or different (25), and size-selection tasks that require attending to both target and distractor by means of a size comparison before selection and response can occur (26).

The referent size-selection procedure has been shown to enhance NP. This procedure requires subjects to compare the semantic referents of two item names, e.g., which of two nouns weighed the most or which of two animal names corresponded to the largest animal $(5,27)$, and has evidenced that NP may also occur when the distractor is processed deeply and reaches awareness. Although the status of a distracting stimulus relative to conscious awareness has not been explicitly described in the inhibition-based model, lack of awareness of the prime's identity has been traditionally one of the markers of NP $(3,16)$. The finding of NP from performance in these decision-type tasks, which favor post-lexical processing in the prime display with overt access to the meaning of both target and distractor, has been considered a challenge to the theory by which NP should result just from earlier inhibition mechanisms in selective attentionpreventing distractors to attain conscious processing. Slowing in response times may be explained by a conflict in the retrieval episode, as a result of ambiguity in information about the target, because the item previously encoded as a distractor is coded as relevant in the current trial and requires a response. The memory-based model predicts more NP when prime selection conditions afford more in-depth processing because the distractor attributes (e.g., identity, spatial location, their status as "relevant" or "irrelevant", and the response "respond" or "do not respond" they require) are likely to be retrievable when the distractor appears as a target in the subsequent trial.

It appears that NP from tasks whose responses are based on identity and location attributes may rely on separate mechanisms (19). Several developmental studies have reported that NP failed to occur in tasks requiring suppression of the identity of distractors in older adults and children, whereas location suppression was spared with age $(12,14)$. These reports from cognitive psychology research were taken as convergent evidence in favor of the existence of at least two separate inhibitory systems involved in identity and location suppression of distractors from visual selective attention tasks, which may be selectively affected across the life span. The hypothesis of a dual nature of the visual system has derived from neurophysiological studies in which two specialized cortical visual pathways that reach the frontal cortex were proposed. The ventral or occipitotemporal pathway seems to be specialized in object shape and in attenuating responses to stimuli previously presented, and the dorsal or occipitoparietal pathway was proposed for processing of spatial location (28).

NP was initially indexed from the total time to name ink-colors in a list of Stroop color-words whose ink-color corresponded to the preceding word that had been recently ignored (2). Experiments recording vocal naming (23) and key-press (29) response latencies to randomized and individually presented Stroop words replicated the effect of distractor suppression, which allowed disregarding the idea that a potential artifact of the massed-list procedure had been the cause of 
slowing in responses. Although the reading list paradigm was criticized because of experimenter effects and insensitivity factors, such as manual operation of a stopwatch and subjects' movements while reading, NP was also reliable in list-reading tasks that required participants to name lists of letters specified by a color cue in letter pairs (30). By including lists of letters alone or paired with unrelated or related distractor letters, increased interference from a concurrent distractor and reliable NP were reported in older adults and adults with Alzheimer's disease (31).

The NP effect on the massed-list procedure from the Stroop color-word task seems to be robust. However, the Stroop task is traditionally implemented in the clinical setting to assess the effect of interference with color naming by the conflicting word, whereas the NP effect derived from the additional cost in naming colors that had been previously ignored, e.g., to name the ink color 'blue' when the previous ignored word had been 'blue', is usually limited to experimental frameworks. While the interference index is good, NP appears to be unreliable and less is known about its psychometric properties (32). As explained above, there has been much debate over the causes of NP, and therefore this effect has been extensively studied in order to clarify its construct validity and to specify which mechanisms may be involved in the effect, in order to allow cognitive psychologists to estimate how the impairment in NP could contribute to the cognitive deficits of several clinical groups and to construct reliable NP indices. This is true for all attempts to implement any NP task as a diagnostic tool, given that NP is usually a small effect which is not reliably observed in the performance of all participants. When the list procedure of the Stroop task is implemented, there is reason to suspect that practice effects across the colorword lists may affect NP scores. In fact, repeated exposure to the Stroop task has been shown to affect the magnitude of the interference by conflicting words with color naming, especially among older subjects. Once the effect of practice was controlled by implementing an additional unrelated colorword list, reliable NP scores could be obtained from the reading-list procedure of the Stroop task in younger and older people (Rosin FM, unpublished data). This procedure is also relatively fast and inexpensive, but it relies on reading ability, so that the measures of performance of people of low educational level and children are usually affected. Also, it cannot be administered to color-blind people.

In the present study, a digit-comparison task was developed in a paper-and-pencil version in order to obtain a reliable measure of NP in a practical way. This task involves the referent size-selection procedure (i.e., subject is asked to compare pairs of items and select the target specified by a semantic cue), ensuring that both target and distractor are attended, since this procedure has been demonstrated to enhance NP. The selection of digits as stimuli obeyed to the fact that the task was developed with the aim to be administered in a clinical setting. In fact, at the time a simultaneous study with patients with cerebral damage was being carried out. Some of them were non-readers or with low degree of formal education, and from heterogeneous cultural background. So, it was more difficult to employ stimuli with highest semantic weight, and letter stimuli were not suitable for the assessment of the whole sample of patients. By contrast, all patients knew digits and were able to perform the paper-and-pencil digit-comparison task. In this task, subjects are asked to circle the target, which reduces somewhat other motor activities that could affect the measure in a reading-list procedure and enables more accurate assessment of errors. The stimulus pairs are digit-asterisk and digit pairs. The task requires circling the digit of the digitasterisk or the greater of two digits in a series of stimulus pairs listed on a sheet of paper. 
Digit stimuli were selected in order to develop a suitable task for the clinical setting, ensuring that people with different educational backgrounds would know the stimuli and could manage them. There are three separate lists: the first is the asterisk-digit condition (asterisk list), in which the distractor is a non-digit stimulus and remains the same throughout the list; the second is the unrelated digit-pair condition (unrelated list) in which distractor and target digits, in successive digit pairs, are never the same; and the third list is the related digit-pair condition (related list), in which in some pairs the distractor in one digit pair is the target in the next pair. Figure 1 shows examples of the stimuli. Marking times are recorded for each list. The asterisk list serves as a baseline measure to estimate the additional time taken for selection between the digits. The slowing in marking time for the related list relative to the unrelated list condition is an index of NP.

The first experiment was run as a pilot demonstration to assess whether the paperand-pencil digit-comparison task indexed NP and whether the NP measure was affected by the order of administration of the lists. The second experiment included the asterisk

\begin{tabular}{|c|c|c|c|c|c|c|c|c|c|c|c|c|c|c|}
\hline \multicolumn{3}{|c|}{ Asterisk list } & \multicolumn{6}{|c|}{ Unrelated list } & \multicolumn{6}{|c|}{ Related list } \\
\hline * 9 & * 7 & $* 8$ & 8 & 7 & 7 & 5 & 8 & 4 & 8 & 9 & 5 & 7 & 8 & 7 \\
\hline $5 *$ & $* 8$ & $* 9$ & 4 & 5 & 9 & 8 & 9 & 5 & 7 & 8 & 4 & 5 & 7 & 5 \\
\hline $9 *$ & $7 *$ & $* 7$ & 8 & 9 & 4 & 7 & 7 & 4 & 7 & 4 & 9 & 8 & 5 & 4 \\
\hline$* 8$ & $8 *$ & $8 *$ & 5 & 4 & 5 & 8 & 5 & 8 & 5 & 9 & 5 & 8 & 7 & 9 \\
\hline $7 *$ & $* 9$ & $7 *$ & 7 & 9 & 9 & 4 & 4 & 9 & 5 & 4 & 4 & 5 & 7 & 5 \\
\hline $8 *$ & $5 *$ & $* 9$ & 4 & 8 & 7 & 5 & 8 & 7 & 9 & 7 & 8 & 9 & 5 & 4 \\
\hline
\end{tabular}

Figure 1. Examples of the stimuli used in the digit-comparison task. In the digit-asterisk condition, the distractor is a non-digit stimulus and remains the same throughout the list. In the unrelated condition, distractor and target digits vary in successive digit-pairs. In the related condition, the distractor digit in a pair is the same as the next target. The asterisk and unrelated lists were preceded by a sample sheet to ensure that subjects had understood the instructions. The time to complete each list and errors were recorded. The asterisk condition was considered to be a baseline measure of performance. The negative priming effect was indexed from the difference in marking times between the unrelated list and the related list. list, which provided a baseline measure of speed of performance in a simpler condition, representing a control measure to estimate the difference in performance when comparison between conflicting digits was required. In addition, performing the asterisk condition may have included initial practice effects on marking times. This experiment tested the effect of practice on the time to complete the unrelated and related lists by adding an additional unrelated list after the unrelated and related lists were completed.

A third experiment was carried out to determine whether aging affects NP as assessed by the paper-and-pencil digit-comparison task, and included a computer-implemented version of the digit-comparison task with recording of vocal naming response latencies to randomized and individually presented stimulus pairs. This experiment also included a target location-based task to assess the possibility that older adults might be able to show NP when the location of the target was the focus of the response, but not when its identity was the basis of selection and response.

\section{Experiment 1}

\section{Method}

Participants. Thirty-three university students, 17 women and 16 men (range $=18-31$ years, mean $=20.52$, , participated in this study. All participants had normal or corrected-to-normal near visual acuity as measured with an ophthalmic vision tester (Standard number 1, Bausch \& Lomb Occupational Vision Tests, New York, NY, USA). All participants gave informed consent to participate in the study which was approved by the Ethics Committee of the Hospital das Clínicas, Faculdade de Medicina de Ribeirão Preto, USP (Project HCRP No. 2825/98).

Experimental design. Participants performed individually the paper-and-pencil digitcomparison task, which required to use a felt 
pen to circle the digit of digit-asterisk pairs or the greater digit of two digits in a series of stimulus pairs listed on a sheet of paper. Separate lists (unrelated and related lists) contained 54 stimulus pairs each, which were distributed across three columns laid out on A-4 size sheets (see example of stimuli for unrelated and related lists in Figure 1). Stimuli in a pair were spaced $1 \mathrm{~cm}$ apart and pairs down the list were spaced $1 \mathrm{~cm}$ apart. Columns were spaced approximately $4.7 \mathrm{~cm}$ apart. Digits were $4 \mathrm{~mm}$ high x $2 \mathrm{~mm}$ wide bold font characters. Five disyllabic (for the Portuguese language) digits were used for all conditions, i.e., 4, 5, 7, 8, and 9. Target and distractor locations, i.e., to the left or right in a pair, were evenly balanced and randomly distributed. The digit 4 appeared as a distractor more frequently than other digits and the digit 9 appeared as a target more frequently than other digits. Target and distractor locations across pairs of successive trials were equally distributed across lists.

Participants completed a sample sheet to ensure that they had understood the instructions and then, they completed the unrelated and related lists. The unrelated list contained unrelated digit pairs in which target and distractor digits, in successive pairs, were never the same. The related list consisted of 20 unrelated digit-pairs and 34 related digit-pairs, i.e., the distractor in one digit pair was the target in the next digit pair. Eighteen participants performed the unrelated list first and 15 participants performed the related list first. Eight participants were asked to complete an additional unrelated list at the end of the task to assess additional practice effects.

Participants were shown the sample and told to circle the greater digit of the digit pairs for the lists, as fast as possible. Subjects were also told that if they made a mistake they should correct it by crossing out the wrong digit and circling the correct digit. A stopwatch was used to measure marking times. For each list, the experimenter said "Ready"... "Go", and started the stopwatch on the word "Go", stopping it as the subject circled the last digit. To avoid possible experimenter effects, a neutral experimenter that ignored the order of the list conditions recorded the marking times. Errors and omissions were also recorded. The number of errors showed to be minimal in healthy young people and they tend to correct them on the go. However, a simultaneous study revealed an increase in errors and omissions in the performance of some patients with cerebral damage. Since speed of performance may hide a differential cost in accuracy across subjects, a formula that takes into account omissions and the commission of errors that were not spontaneously corrected could be useful to obtain a more accurate measure of performance when comparisons betweengroups are required. For that purpose, it was estimated the corrected marking time (cMT) for all list conditions, which was computed as follows: $\mathrm{cMT}=$ (marking time/total of pairs marked) x (non-corrected errors + omissions) + marking time. In the present study, conducted on healthy young and older people, all analyses carried out on the cMTs yielded similar pattern of significant effects to the analyses conducted on the marking times without the inclusion of errors, so this last and most simple measure was used.

In order to assess NP, i.e., the delay to complete the related list relative to the unrelated list, the marking times for each subject on lists in each condition were submitted to analysis of variance (ANOVA), with repeated measures for the list conditions. Duncan's multiple range test was used for post hoc comparisons. Wilcoxon's matchedpair test was used for within-subject comparison of mean errors and Mann-Whitney's U-test was used for between-subject comparisons. An alpha level of 0.05 was used for all statistical tests.

\section{Results and Discussion}

Figure 2 shows the mean marking times 
for the unrelated and related lists according to the order of presentation of the lists. A 2 (list condition: unrelated $v s$ related) $\mathrm{x} 2$ (order of list) ANOVA revealed that subjects were slower in completing the related list, with digit targets that had been previously ignored as distractors, than they were in completing the list with unrelated digit pairs, $\mathrm{F}(1,31)=$ $15.71, \mathrm{P}=0.0004$. These higher marking times for the related condition indicated reliable NP. The main effect of list order was absent, $\mathrm{F}(1,31)=0.36, \mathrm{P}<0.551$, but the interaction between order and list was significant, $\mathrm{F}(1,31)=6.16, \mathrm{P}<0.019$. Post hoc comparisons revealed that the marking times for the related list were significantly slower than for the unrelated list, but only in the group that had performed first the unrelated list test, as can be seen in Figure 2. However, the time taken to complete the related list presented first was equivalent to that observed when the related list was completed after the unrelated one. Thus, the order of list presentation seemed to affect just the unrelated condition, with higher marking times when the unrelated list test was performed after the related list.

ANOVA conducted on the marking times of the 8 participants that completed the unrelated - related - additional unrelated lists, revealed that list condition was significant, $F(2,14)=9.74, P=0.002$. A reliable slowing for the related condition was observed relative to both first and additional unrelated lists,

Figure 2. Effect of the order of administration of the lists on the difference in marking time between the unrelated ( $U$ list) and related ( $\mathrm{R}$ list) conditions, in Experiment 1. Data are reported as the mean \pm SEM. Eighteen participants performed the $U$ list first, and 15 participants performed the $\mathrm{R}$ list first. Participants were significantly slower in completing the related list when they had performed the unrelated list before, ANOVA (2 list $\times 2$ order), $F(1,31)=6.16, P$ $<0.019$, followed by Duncan's multiple range test ${ }^{*}(P<0.01)$. tional unrelated $(\mathrm{M}=42.61, \mathrm{SD}=9.09)$, as indicated by post hoc multiple comparisons.

Analyses of errors did not yield significant differences across list conditions or groups. The error means, $0.09(\mathrm{SD}=0.38)$ for the unrelated list and $0.18(\mathrm{SD}=0.46)$ for the related list, were not significantly different, $\mathrm{T}$ $=3.00, \mathrm{Z}=1.21, \mathrm{P}=0.225$. Groups did not differ from one another in errors for the unrelated list, $\mathrm{U}=133, \mathrm{Z}=-0.07, \mathrm{P}=0.942$, or related list, $\mathrm{U}=129, \mathrm{Z}=-0.20, \mathrm{P}=0.842$.

Finally, a two-way ANOVA ( 2 sex x 2 list condition) revealed that men were overtly faster than women, $\mathrm{F}(1,31)=8.06, \mathrm{P}<$ 0.008 ; both groups were slower for the related condition, $\mathrm{F}(1,31)=14.77, \mathrm{P}=$ 0.0006 , with no interaction between sex and list condition, $\mathrm{F}(1,31)=0.01, \mathrm{P}=0.943$. $\mathrm{NP}$ was reliable for both the groups of men, $\mathrm{F}(1$, $15)=7.14, P=0.017$, and women, $F(1,16)$ $=7.58, \mathrm{P}=0.014$. Since women showed an overall slowing in response times, ANOVA was conducted on the proportional NP measure, i.e., [(marking time for related list minus - marking time for unrelated list)/ marking time for unrelated list] which did not yield significant difference in proportional NP between sex groups, $\mathrm{F}(1,31)=0.09, \mathrm{P}$ $=0.767$.

The digit-comparison task seemed to reflect NP, as indicated by slower times to complete the related list, with recently ignored digits, compared to the time taken to complete the unrelated list with different digits in successive digit-pairs. The order of the list conditions affected the NP index, i.e., the difference between the unrelated and related conditions was absent when the related list was completed first. However, this effect may not be explained by an improvement in time related to repeated performance of lists, since the reliable slowing for the related list occurred just when this was completed after practice on the unrelated list. Furthermore, differences in marking times for the related condition were not detected between groups with inverted order of the 
lists (see Figure 1). This result addressed the concern of whether practice and possible attentional strategies that could develop across trials, also including motor practice (and/or fatigue), could have influenced performance indices and consequently affected the NP measure. For the group of 8 participants that completed the final unrelated list there was a trend towards an increase in marking time for the unrelated condition after a prior exposure to the related list, although the difference did not reach significance, and a reliable slowing for the related condition was observed relative to both first and additional unrelated lists.

Therefore, Experiment 2 was carried out to reassess the NP finding by means of the paper-and-pencil digit-comparison task and extended the question of whether practice was affecting differences in marking times across list conditions by requiring all participants to complete an additional unrelated list after the related condition. Additionally, Experiment 2 also included the asterisk list with non-conflicting distractors, which served as a baseline measure of speed of performance and to estimate the additional cost in comparing and selecting between two digits. So, the conditions, in the order in which they were administered to the participants, were the asterisk list, the unrelated list, the related list, and finally, the additional unrelated list.

\section{Experiment 2}

\section{Method}

Participants. Twenty-three university students, 8 women and 15 men (range $=19-38$ years, mean $=23.48$ ) with normal or corrected-to-normal near visual acuity, participated in this study. None had participated in Experiment 1. All participants gave informed consent. The research was approved by the Ethics Committee as cited in Experiment 1.

Material and procedure. Stimuli and procedure were the same as described in detail in Experiment 1. The procedure was otherwise identical to that described above, but included the asterisk condition which required participants to circle the digit of the digit-asterisk pairs. For the unrelated and related lists the asterisk list also contained 54 stimulus pairs distributed across three columns laid out on A-4 size sheets (See Figure 1). For the digit stimuli, asterisks were also bold font characters, but subtending $2 \times 2$ $\mathrm{mm}$. Both asterisk and unrelated lists were preceded by a sample sheet containing eight stimulus pairs to ensure that the subject had really understood the instruction. Participants were told to circle down the digit of the digit-asterisk pairs for the asterisk list, and the greater digit of the digit pairs for the following lists, as fast as possible. The unrelated and related lists were identical to those described in Experiment 1. The asterisk list was the first one and contained digit-asterisk pairs. The second list was the unrelated condition, in which target and distractor digits, in successive digit pairs, were never the same. The third list was the related condition, which contained related digit-pairs, i.e., the distractor in one digit pair was the target in the next digit pair. Finally, all participants completed an additional unrelated list.

As described for Experiment 1, the basic dependent measure was each subject's marking time on lists in each condition. Primarily, NP was evaluated by comparing the marking time in the unrelated condition to the marking time in the related condition. Furthermore, the additional time related to conflicting comparison between digits was also estimated by comparing the marking time in the unrelated condition to the marking time in the asterisk condition.

\section{Results and Discussion}

Table 1 summarizes the data, response times and errors. Significant differences in marking times across asterisk, unrelated, and related conditions were observed, with 
reliable NP. ANOVA was used for comparison of the marking times in the appropriate list conditions (asterisk vs unrelated vs related), which revealed that the effect of condition was significant, $\mathrm{F}(2,44)=52.42$, $\mathrm{P}<0.0001$. As expected, post hoc analysis revealed that participants took more time in completing the unrelated list than the asterisk list. NP was reliable, with slower marking times for the related list than for the unrelated list.

To assess whether practice affected the differences in time taken to complete the list conditions, the marking times were subjected to ANOVA with repeated measures on condition (unrelated $v s$ related $v s$ additional unrelated). The effect of condition was significant, $\mathrm{F}(2,44)=15.09, \mathrm{P}<0.0001$, with longer marking times for the related list indicating reliable NP. Again, post hoc comparisons showed that subjects were slower in completing the related list than in completing each of the two, first and additional, unrelated lists. Furthermore, the marking times did not differ significantly between the two unrelated lists. Planned comparisons also showed a non-significant difference between the unrelated lists, $\mathrm{F}(1,22)=2.33$, $\mathrm{P}<0.141$. Exam of errors showed that they did not differ across conditions. Friedman ANOVA indicated that errors were neither significantly different across asterisk $v s$ unrelated $v s$ related lists, $\chi^{2}(2,23)=1.00, \mathrm{P}<$

Table 1. Marking time and errors for the paper-and-pencil digit-comparison task in Experiment 2.

\begin{tabular}{|c|c|c|c|c|}
\hline & \multirow[t]{2}{*}{ Digit-asterisk } & \multicolumn{3}{|c|}{ Digit-pairs } \\
\hline & & Unrelated & Related & $\begin{array}{l}\text { Additional } \\
\text { unrelated }\end{array}$ \\
\hline Marking time & $34.61 \pm 8.45$ & $42.64 \pm 10.01$ & $46.26 \pm 9.95$ & $43.68 \pm 10.94$ \\
\hline $\begin{array}{l}\text { Errors } \\
\text { Negative } \\
\text { priming (\%) }\end{array}$ & $0.00 \pm 0.00$ & $\begin{array}{r}0.04 \pm 0.21 \\
9.22\end{array}$ & $\begin{array}{l}0.04 \pm 0.21 \\
8.33\end{array}$ & $0.22 \pm 0.85$ \\
\hline
\end{tabular}

Data are reported as means $\pm S D, N=23$, in seconds for marking time, and number of errors.
0.607 , nor different across unrelated $v s$ related $v s$ additional unrelated lists, $\chi^{2}(2,23)=$ $0.50, \mathrm{P}<0.779$.

The results replicated those obtained in Experiment 1, showing reliable NP for the paper-and-pencil digit-comparison task, expressed as a significant delay in completing the related digit-pair list relative to the unrelated list. The results of Experiment 2 do not support the notion that NP should be attributed to practice biases or fatigue effects. The suggestion that such delay may have relied upon expectancy biases for successive stimuli, which may have developed after repeated exposure to successive ignoredrepeated stimuli of the related list, was addressed in this study by completing two unrelated lists, one before and the other after performing in the related condition. In both unrelated lists the marking times were faster than the time taken to complete the related list, with no significant differences between them. These results disagree with the claim that slowing in a related digit-pair list may be explained by expectancy biases developed after repeated exposure to recently ignored digit stimuli.

Additionally, this study included an asterisk list containing non-conflicting distractors, a less demanding list than the digit-distractor list. The asterisk list serves to obtain a baseline marking time, also permitting betweensubject comparisons of proportional additional cost in comparing and selecting between two digits, as done in Experiment 3.

\section{Experiment 3}

This study included younger and older adults in order to determine whether NP measurement, as assessed by means of the paper-and-pencil digit-comparison task, was affected by aging. Several findings provided evidence that older adults do not show consistent slowing for recently ignored items across different NP tasks and under the same experimental circumstances as do younger 
adults $(6,13)$. Although contradictory experimental outcomes also exist $(10,31)$, older adults seemed not to demonstrate reliable NP primarily in identity NP tasks, which require a response to the identity of a target $(13,14)$. In contrast, NP seemed to be preserved in aging when the task required a response to the location of the target (12). Stronger evidence of a dissociation between identity and spatial NP was obtained in the present experiment, in which younger, middle-aged and older adults were tested on both identityand location-based NP tasks.

Although the present version of the digitcomparison task could combine some aspects of the spatial component with identification of the target, since the target appears sometimes at the same site as the disregarded smaller digit in the previous digit-pair, the design of the task was thought to focus on semantic aspects of the target for both selection and response rather than on its localization. The instruction explicitly oriented participants to mark the larger digit, and did not include any mention to site-related features. Furthermore, the site alternatives were very reduced - just two -, and there was no fixation point or a spatial distribution that would allow to organize the visual field into hemifields. Actually, the spatial type of NP could involve some difficulties to be detected by means of typical list procedures; however, it would be very practical for clinical assessment in order to develop a paper-andpencil version of a site-based NP task. Preliminary results of a pilot test in which a version of the site-based ' $O X$ ' task was implemented in a paper-and-pencil design with a massed-list procedure did not demonstrate a particular slowing for targets imprinted at the previously ignored sites (Rosin FM, unpublished results). In any case, the potential site-based effect could be mixed with identity-based processes in the responses to the digit-comparison task. Thus, besides the paper-and-pencil digit-comparison task, middle-aged and older participants performed on a computerized version of the digit-comparison task in which the identity was the attribute overtly reported. In the computerimplemented digit-comparison task, they were asked to name aloud the digit target that appeared in digit-pairs on a screen display. Then, participants performed a modified version of the 'OX' spatial NP task such that the target location was the reported attribute (24).

Since older participants exhibited slowing in response times for all tests and proportional (ratio) scoring has been proposed in previous studies of NP as a more conservative method for comparisons between groups with different baseline in response times (8), in this study we included proportional measures of NP in data analysis to compare group effects. Analysis of the proportional effects on response times has been proposed because slowing usually tends to magnify absolute differences between conditions, and ANOVA performed on the difference in scores between groups with different baseline response times - or across tasks that differ in their respective baseline measures - will often show significant group interaction effects despite exhibiting similar proportional differences in response times. Using proportions rather than absolute differences could continue to be debated because converting differences to proportions of baseline for each group would yield diminished NP for subjects who respond in an overall slower manner than for their corresponding control groups. As pointed out, a method which assumes a proportional relationship in the response times accounts for the general slowing problem that is closely involved in measuring specific attentional effects not only in aging subjects, but also in patients with brain damage $(8,9)$, and proportional scoring would provide a more accurate depiction of performance than the alternative of examining only absolute difference scores. While proportional scoring may fail to be accurate enough to capture the complexity 
of an effect if there is differential slowing of specific processes underlying task performance, it may be appropriate if the assumption is made that all processes underlying task performance are slowed uniformly. Developmental studies specifically targeting this problem have shown that a global, not local, slowing may affect information processes underlying performance in memory and selective attention tasks in older people (33). This is a reason to emphasize the importance of first including a measure that differentiates between individuals with potentially different baselines in their response latencies when more specific attentional measures should be assessed. Then, it is possible to compare gross performance on a more basic level of processing and to test whether there exist group effects on increases in latency, and to contrast overall slowing effects of increasing complexity of the task with process-specific effects. Furthermore, meta-analyses conducted from several developmental studies provided evidence to support the view that NP is proportional rather than additive, with diminished NP in older adults as compared with younger adults (34).

In the present study the possible disproportionate effects on response times were assessed when age group performance differed in the simplest condition, i.e., when the target was paired with a non-conflicting stimulus (asterisk) or when it was presented without distractors in one of the four possible locations on the site-based NP task. Then, the cost for the condition of increasing complexity of the task was estimated besides the experimental effect of NP. Moreover, central conclusions were derived in a more conservative way, based on patterns of performance that are qualitatively similar when estimated as absolute or proportional differences.

Other question that the present experiment addresses is the age-group difference in years of education that could affect NP measures from the paper-and-pencil digitcomparison task. Although this task was specially designed to not be affected by education, the Experiment 3 reassessed NP from the paper-and-pencil task with younger participants that were university students, and with middle aged and older adults with equivalent years of education.

\section{Method}

Participants. Eight younger adults, three women and five men (range $=18-24$ years, mean $=21.38$ ), eight middle-aged adults, five women and three men (range $=31-54$ years, mean $=41.00)$, and eight older adults, three women and five men (range $=68-77$ years, mean $=70.88$ ), participated in this study. The younger adults were undergraduate students and had a mean of $13.38(\mathrm{SD}=1.51)$ years of education. Mini-Mental State Examination (35) was given for the middleaged and older adults to test their general cognitive abilities. The middle-aged adults were recruited from University Campus personnel and had a mean of $8.25(\mathrm{SD}=3.65)$ years of education. The older adults were recruited from several recreation centers where they were actively involved; they were selected from a larger sample in order to be matched to the middle-aged individuals in terms of years of education and MiniMental Score, and they had a mean of 6.75 $(\mathrm{SD}=3.20)$ years of formal education. None of the participants reported histories or signs of neurological or psychiatric diseases and all had normal or corrected-to-normal near visual acuity required to perform the tasks. All subjects gave informed consent to participate in the investigation and none of them participated in more than one study. The research was approved by the Ethics Committee as cited in Experiment 1.

Since performance of tasks supposed to demand attentional inhibitory processes seems to be influenced by circadian rhythms in aging (36), the best circadian peak of each 
older participant was considered when planning the schedule of the experimental sessions by applying the Morningness-Eveningness Questionnaire (37), which was applied to the participants by telephone. Three older participants were definitely morning types and five older participants were moderately morning types.

Procedure. Participants were assessed individually. Younger participants completed the paper-and-pencil digit-comparison task. The Mini-Mental State Examination (version traduced and revised by Bertolucci PHF, Universidade Federal de São Paulo, SP, Brazil) was given to the middle-aged and older adults to test their general cognitive abilities. The experimental tasks, in the order in which they were administered to the middle-aged and older participants, were the paper-andpencil digit-comparison task, the computerized version of the digit-comparison task, and the computerized 'OX' local-based task. For the computerized tasks, testing took place in a quiet and dimly lit room and participants sat in a chair with adjustable height, with their heads standing approximately $60 \mathrm{~cm}$ from the center of the screen.

\section{Paper-and-pencil digit-comparison task}

Material and procedure were identical to those described in Experiment 2, except that the subject did not complete the additional final unrelated sheet. Thus, there were three list conditions (asterisk $v s$ unrelated $v s$ related), and the marking times for each list were the critical measures to indicate the baseline of speed of performance and to estimate NP.

\section{Computerized digit-comparison task}

An additional measure of NP was obtained from a digit-comparison task that was programmed using the MEL Professional V2.01 software (38). In all trials, subjects named aloud the digit of a digit-asterisk pair or the greater digit of a digit-pair. Stimulus pairs were presented binocularly on a color dual scan display $(16 \mathrm{~cm} \times 21 \mathrm{~cm})$ of an IBMcompatible notebook computer elevated 20 $\mathrm{cm}$ from the desk and equipped with a serialresponse box (Psychological Software Tools, PA, USA). A voice key interface was used to measure naming latencies. Response timing was measured at $1-\mathrm{ms}$ resolution.

Digits (from 0 to 9) measured 4 (width) x 8 (height) $\mathrm{mm}$, subtending approximately $0.39^{\circ} \times 0.76^{\circ}$ of the visual angle; and the asterisk measured $5 \times 6 \mathrm{~mm}$, subtending approximately an angle of $0.48^{\circ}$ horizontally and of $0.57^{\circ}$ vertically. The fixation point was a plus sign, subtending approximately $0.57^{\circ} \times 0.57^{\circ}$ vertically $(6 \times 6 \mathrm{~mm})$ and was centered on the screen. The font type was of the 'system56.fnt' graphics of Professional MEL2. Pairs of stimuli were centered on the screen with approximately $1.7 \mathrm{~cm}$ separating their nearest edges. The color of the stimuli was white and the stimuli were shown against a dark background.

There were three trial conditions corresponding to the three levels of the withinsubjects factor: asterisk trial, which presented a digit-asterisk pair; unrelated trial, which presented a digit-pair with digits in the previous trial different from digits in the current trial, and related trials with a digit target identical to the distractor in the previous trial. Trial conditions were randomly distributed. There were 50 asterisk trials, 54 unrelated trials, and 59 related trials. Target and distractor locations, i.e., to the left or to the right of the fixation, were evenly balanced and varied randomly from trial to trial. Subjects responded to the target in a fixed ongoing sequence of trials. Thus, the stimuli that acted as a probe in a particular trial also acted as a prime in the next trial. Each trial proceeded as follows: a fixation cross consisting of a $6 \times 6 \mathrm{~mm}$ plus sign (+) centered on the computer screen for $250 \mathrm{~ms}$, a blank screen for $250 \mathrm{~ms}$, and the display containing a digit-asterisk pair or a pair of digits which 
remained visible until the participant made an oral response. Then, the cross fixation reappeared for $250 \mathrm{~ms}$ for the next trial, and so forth. There were two first practice blocks, each consisting of five trials, and three experimental blocks consisting of 67,73 and 76 trials, respectively. Practice trials, the first trial for each experimental block, and trials following asterisk trials were not included in data analysis.

Subjects were encouraged to rest between blocks. Each block was initiated by the experimenter by pressing the space bar. The naming latencies were measured as the interval between the stimulus onset and the participant's vocal response into a microphone. Accuracy was recorded by the experimenter on a response list. Both speed and accuracy were emphasized, but subjects were told that if they made a mistake they should continue with the digit naming task rather than trying to correct it.

Error trials, spoiled trials when the participant's response failed to trigger the microphone or when it was triggered prior to $100 \mathrm{~ms}$, and trial immediately following an error were excluded from the naming-latency analysis of the data. The dependent variables were median naming latencies and error percentages by trial condition. The naming latencies for asterisk trials provided a baseline measure of performance. NP was assessed by comparing the median naming latencies for the unrelated trials to the naming latencies for the related trials.

\section{Location-based priming task}

One spatial measure of NP was obtained from a computerized task designed on the basis of the 'OX' task for location-based NP (24) using the MEL Professional V2.01 software to record reaction times, with accuracy of up to $1 \mathrm{~ms}$ (38). In all trials, subjects indicated the location of a target (the letter ' $\mathrm{O}$ ') appearing, alone or with a distractor (the letter ' $X$ '), at one of four locations by pressing the key corresponding to the location. Stimuli were presented on an IBMcompatible notebook computer, which was elevated $20 \mathrm{~cm}$ from the desk and equipped with a serial-response box (Psychological Software Tools) with five buttons and lamps (rightmost button and lamps were covered) aligned with the screen.

The four locations were four horizontal bars $(4 \times 1.5 \mathrm{~mm})$ centered on the screen, aligned in a row subtending approximately $6.4^{\circ}$ of the visual angle $(67 \mathrm{~mm})$ between the two outside positions, and remained on the screen throughout all displays marking four positions. The fixation point was a plus sign, subtending approximately $0.48^{\circ}$ horizontally and $0.39^{\circ}$ vertically $(5 \times 4 \mathrm{~mm})$, and was displayed $7 \mathrm{~mm}$ above the row of locations and between the second and third location. The target ' $\mathrm{O}$ ' and distractor ' $\mathrm{X}$ ' (upper case letters of 'system56.fnt' graphics MEL2 font type) subtended approximately $0.86^{\circ}$ vertically and $0.43^{\circ}$ horizontally $(9 \times 4.5$ $\mathrm{mm}$ ). The color of the stimulus was white and the stimulus shown against a dark background.

Participants responded to the target, while ignoring the distractor, if present, in a fixed ongoing sequence of trials. Thus, the position of the ' $\mathrm{O}$ ' that was the probe on a particular probe trial also acted as the prime in the next trial. There were three types of trial conditions: target-alone, unrelated target-plus-distractor and related target-plusdistractor, which were randomly distributed. In both target-alone and unrelated trials, the stimuli reappeared at locations that were not occupied in the preceding prime trial. In related trials, the target occupied the same location the distractor had occupied in the preceding prime trial. The dependent variables were median response times and percent errors for each trial condition. Two types of effects were observed: the interference of the distractor and NP. The measure of interference was derived by comparing the response times for target-alone trials with 
those for unrelated trials. The difference in response time between these two types of trials was a measure of the extent to which the presence of a distractor interfered with responses to the location of the target. NP was assessed by comparing the time to respond to unrelated trials with the time to respond to related trials. The difference in response time between these two types of trials was a measure of spatial NP.

Trials were divided into five blocks: two first practice blocks consisting of six and five trials, respectively, and three experimental blocks consisting of 44, 49, and 47 trials, respectively. Locations of target and distractor were evenly balanced. There were 37 related trials, 37 unrelated trials, and 32 target-alone trials. The first trial of each block, two buffer trials, 29 trials following target-alone trials, error trials and the trial immediately following an error trial, and response made prior to $150 \mathrm{~ms}$ following target-display presentation were not included in the response time analysis of the data.

Participants were shown the stimulus display and the four keys of the serial box and were asked to fixate on the fixation point while it was activated and to press the response key corresponding to the ' $\mathrm{O}$ ' location. All participants were instructed to use the middle and index fingers of the left hand to indicate the left locations, and the middle and index fingers of the right hand to indicate the right locations. Participants were asked to ignore the ' $\mathrm{X}$ ' as it was irrelevant to the task and to try to be as quick and accurate as possible in responding to the location of the ' $\mathrm{O}$ '. The experimenter pressed the space bar when the participant was ready to resume. Each trial began with the onset of the four location markers. After $500 \mathrm{~ms}$, a fixation cross was presented and left on the screen for $800 \mathrm{~ms}$. The target, alone or presented with the distractor, followed the offset of the fixation cross and remained on the screen until a response was made. After the subject responded to the display, another sequence delay-fixation-display was reinitiated, and so forth. There was no feedback for correct responses. A 0.5 -s tone $(400 \mathrm{~Hz})$ signaled the incorrect responses.

\section{Results and Discussion}

\section{Subject comparisons}

As previously stated, the group of younger student participants had more years of education than did the older and middle-aged groups, $\mathrm{U}=3.00, \mathrm{Z}=-3.05, \mathrm{P}=0.002$. Older and middle-aged participants did not differ in their years of education, $U=26.0, Z=-0.63$, $\mathrm{P}=0.650$, or their Mini-Mental Scores, $\mathrm{U}=$ $19.0, \mathrm{Z}=-1.37, \mathrm{P}=0.172$, with a mean score $($ Max score $=30)$ of $28.38(\mathrm{SD}=1.06)$ for the older group and of $29.13(\mathrm{SD}=0.83)$ for the middle-aged group.

The alpha level for all analyses was $\mathrm{P}<$ 0.05 . The data were first subjected to ANOVA with age as between-subject factor and condition as within-subject factor, with repeated measures of conditions. This analysis was followed by Duncan's multiple range test for post hoc comparisons. Since older participants exhibited a different baseline in response times, with overall slowing for all tasks, and since a proportional (ratio) scoring has been proposed as a more conservative way for comparisons between groups with different baseline in response times (8), proportional measures were also used to compare group effects. Thus, proportional scoring relative to the appropriate control condition was computed for group comparisons and across-task comparisons.

\section{Paper-and-pencil digit-comparison task}

Table 2 summarizes the mean marking times and errors for each list condition by age group. Errors were infrequent (14 in the whole experiment) and did not differ significantly across age groups or types of list. Percent errors made in each list condition for 
each subject were subjected to separate Friedman ANOVA, with repeated measures on condition, and Kruskal-Wallis ANOVA with age as between-subject factor, both of which yielded non-significant effects. The difference in errors across list conditions was not statistically significant for younger participants, $\chi^{2}(8,2)=4.00, \mathrm{P}=0.135$, or middleaged participants, $\chi^{2}(8,2)=4.67, \mathrm{P}=0.097$, or older adults, $\chi^{2}(8,2)=3.50, \mathrm{P}<0.174$. Age groups did not differ in their errors for asterisk, related, or unrelated lists, $\mathrm{H}(2,24)$ $=0.00, \mathrm{P}=1.00$. Related and unrelated lists were the critical conditions for measuring NP. The data were first subjected to 3 (age: younger $v s$ middle-aged $v s$ older) $\mathrm{x} 3$ (condition: asterisk $v s$ unrelated $v s$ related) ANOVA. This analysis revealed that older adults were slower than younger and middle-aged adults, $\mathrm{F}(2,21)=4.67, \mathrm{P}=0.021$. List condition was significant, $\mathrm{F}(2,42)=104.05, \mathrm{P}<$ 0.0001 , with the following profile in marking times: asterisk $<$ unrelated $<$ related, indicat- ing reliable NP. The age $\mathrm{x}$ condition interaction was not significant, $\mathrm{F}(4,42)=0.93, \mathrm{P}<$ 0.454 .

Further ANOVA tests were first applied to the absolute differences in performance across lists. Two effects were observed: the cost in speed of performance due to the difference in complexity of the required cognitive operations between the digit-pairs and asterisk-digit conditions (unrelated marking time minus asterisk marking time), and NP (related marking time minus unrelated marking time). Groups did not differ in their costs to complete the digit-pair condition relative to the easier asterisk-digit condition, $\mathrm{F}(2,21)=$ $0.15, \mathrm{P}=0.863$. Differences in the cost to complete the related condition as expressed by absolute differences were not statistically significant across groups, $\mathrm{F}(2,21)=2.77$, $\mathrm{P}$ $=0.086$. However, planned comparison analysis indicated that the differences between related and unrelated lists were significantly smaller for the older group than for the

Table 2. Response time and errors for conditions across tasks by age groups in Experiment 3.

\begin{tabular}{|c|c|c|c|}
\hline & Younger adults & Middle-aged adults & Older adults \\
\hline \multicolumn{4}{|c|}{$\begin{array}{l}\text { Paper-and-pencil digit-comparison task } \\
\text { (response time in seconds) }\end{array}$} \\
\hline Asterisk & $31.47 \pm 5.48(0.00 \pm 0.00)$ & $37.63 \pm 8.13(0.00 \pm 0.00)$ & $49.20 \pm 9.60(0.00 \pm 0.00)$ \\
\hline Unrelated & $46.33 \pm 11.44(0.00 \pm 0.00)$ & $51.17 \pm 9.15(0.46 \pm 1.31)$ & $62.41 \pm 13.22(0.23 \pm 0.65)$ \\
\hline Related & $52.00 \pm 13.17(0.25 \pm 0.46)$ & $56.73 \pm 9.91(0.50 \pm 0.76)$ & $64.09 \pm 14.36(0.63 \pm 1.06)$ \\
\hline Unrelated-asterisk & $14.85 \pm 7.41$ & $13.53 \pm 5.21$ & $13.20 \pm 6.38$ \\
\hline Related-unrelated & $5.68 \pm 3.67$ & $5.57 \pm 3.06$ & $1.68 \pm 4.70$ \\
\hline \multicolumn{4}{|c|}{$\begin{array}{l}\text { Computerized digit-comparison task } \\
\text { (naming latencies in milliseconds) }\end{array}$} \\
\hline Asterisk & - & $593.69 \pm 65.74(0.00 \pm 0.00)$ & $657.75 \pm 92.83(0.00 \pm 0.00)$ \\
\hline Unrelated & - & $620.13 \pm 62.10(0.95 \pm 1.76)$ & $752.19 \pm 102.03(2.29 \pm 0.62)$ \\
\hline Related & - & $635.69 \pm 71.65(2.52 \pm 1.99)$ & $721.13 \pm 103.49(3.93 \pm 0.85)$ \\
\hline Unrelated-asterisk & - & $26.44 \pm 19.50$ & $94.44 \pm 34.89$ \\
\hline Related-unrelated & - & $15.56 \pm 18.38$ & $-31.06 \pm 22.52$ \\
\hline \multicolumn{4}{|c|}{$\begin{array}{l}\text { Computerized local-based task } \\
\text { (response time in milliseconds) }\end{array}$} \\
\hline Target-alone & - & $591.56 \pm 77.01(0.00 \pm 0.00)$ & $710.81 \pm 127.15(3.52 \pm 6.53)$ \\
\hline Unrelated & - & $616.69 \pm 67.43(1.50 \pm 1.60)$ & $742.50 \pm 123.07(5.09 \pm 3.77)$ \\
\hline Related & - & $632.56 \pm 83.85(3.25 \pm 4.39)$ & $765.38 \pm 122.92(4.73 \pm 3.64)$ \\
\hline Unrelated-target alone & - & $25.13 \pm 22.02$ & $31.69 \pm 30.47$ \\
\hline Related-unrelated & - & $15.88 \pm 21.43$ & $22.88 \pm 48.39$ \\
\hline
\end{tabular}

Response time and its units are given for each task. Errors reported as percent are given in parentheses. Data are reported as means \pm SD for the paper-and-pencil task and mean of medians $\pm \mathrm{SD}$ for the other two tasks. $\mathrm{N}=8$ participants in each group. 
younger groups, $\mathrm{F}(1,21)=5.53, \mathrm{P}<0.029$.

Since the groups exhibited different baselines in response times, statistical analyses were also conducted on proportional scorings to compare group effects. Thus, the proportional (ratio) increase in marking times for the unrelated list relative to the asterisk condition was estimated as follows: digit-comparison cost $=[$ (unrelated) - (asterisk)]/(asterisk); and the proportional increase in marking times for the related list relative to the unrelated condition was computed as follows: proportional NP $=[$ (related) - (unrelated)]/(unrelated). These estimates were submitted to separate one-way ANOVA, with age as random between-subject factor. The proportional scores demonstrated effects that shared the same tendencies identified with absolute difference scores. The analyses revealed that proportional differences in the cost to compare between digits relative to the baseline measure of performance in the asterisk condition were not statistically significant among groups, $\mathrm{F}(2,21)=3.12, \mathrm{P}=0.065$. In contrast, the effect of age on the specific proportional measure of NP was statistically significant, $\mathrm{F}(2,21)=4.76, \mathrm{P}<0.02$, with diminished relative slowing on the related list for older adults relative to both groups of middle-aged and younger participants (see Figure 3).

Because specific predictions were made and a significant difference in NP for older participants was observed, planned comparisons were used to explore the differences among list condition means for the different age groups. NP from the paperand-pencil digit-comparison task, reported as increased time to complete the related list compared to the unrelated list, was evident in the middle-aged group, $\mathrm{F}(1,21)=16.56$, $\mathrm{P}$ $<0.0006$, and younger group, $\mathrm{F}(1,21)=$ $17.21, \mathrm{P}<0.0005$, with no significant differences between them, $\mathrm{F}(1,21)=0.03, \mathrm{P}=$ 0.956 . NP was not observed in older adults, $\mathrm{F}(1,21)=1.51, \mathrm{P}=0.233$, in agreement with previous studies that suggested a failure in identity suppression with aging. This study also shows that the observable NP in young people seems to be reliable during middle-age adulthood. In addition, this result makes it extremely unlikely that the NP measured by the paper-and-pencil digit-comparison task is compromised by years of education.

Computerized tasks. Mean percentage of errors and mean of median naming latencies and response times calculated after excluding error and spoiled trials, for the three trial conditions by age group and the absolute differences between trial conditions for each task are reported in Table 2. Spoiled trials in the digit-comparison task averaged 3.41\% $(\mathrm{SD}=1.65)$ for the middle-aged adults and $18.80 \%(\mathrm{SD}=6.13)$ for the older adults. To assess performance in the computerized tasks, the basic ANOVA used throughout was a 2 (age) x 3 (trial condition), with repeated measures of within-subject conditions.

For the digit-comparison task, middleaged adults were faster than older adults, $\mathrm{F}(1,14)=5.04, \mathrm{P}<0.042$; the naming latencies differed across trial conditions, $\mathrm{F}(2$, $28)=64.97, \mathrm{P}<0.0001$, and age interacted with trial condition, $\mathrm{F}(2,28)=18.16, \mathrm{P}<$ 0.0001 .

To interpret the interaction and to compare the cost derived from comparisons between digits relative to selecting in asterisk trials and the magnitude of NP between groups, ANOVA was applied to the absolute

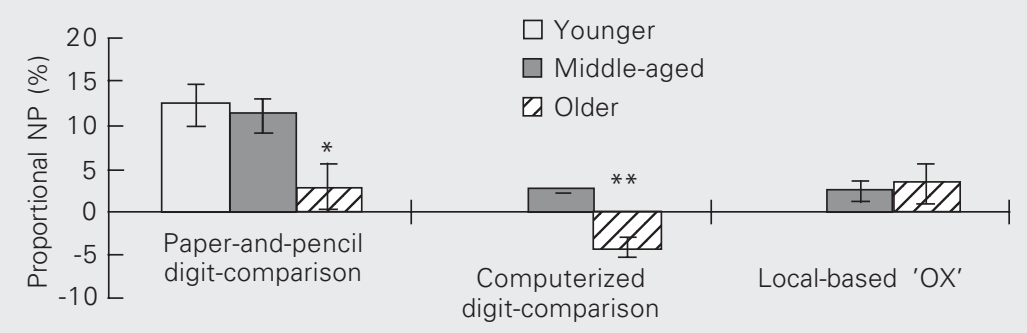

Figure 3. Proportional negative priming (NP) scores for each task in Experiment 3. Data are reported as the mean \pm SEM. NP did not differ across younger and middle-aged participants in the paper-and-pencil digit-comparison task, $F(1,21)=0.03, P=0.956$. The group of older participants showed reduced NP for digit-comparison tasks but preserved local-based NP relative to the NP scores derived from the performance of middle-aged participants; MANOVA (2 age $\times 3$ task), Rao $R(3,12)=16.02, P<0.0002$. Based on Tukey's HSD * $(P<0.05)$ and ${ }^{* *}(P<0.001)$. 
differences in performance across lists. Similar analyses of the proportional scorings: cost in the comparison-task $=$ (unrelated asterisk)/unrelated, and NP = (related - unrelated)/unrelated yielded a similar pattern of significant results. Older adults showed more impairment in their performance in the conflicting digit-pair condition than middle-aged adults, $\mathrm{F}(1,14)=23.15, \mathrm{P}<0.0003$, but middle-aged adults showed more NP than older adults, $\mathrm{F}(1,14)=20.58, \mathrm{P}<0.0005$. Note in Table 2 that the difference between the related and unrelated conditions in older adults had a positive sign, indicating a reversed NP effect rather than slowing for recently ignored targets.

Error data were analyzed by the Wilcoxon matched pairs test applied to each of the effects of interest, i.e., the difference between the unrelated and asterisk conditions and NP, and age groups were compared by the Mann-Whitney U-test. For the digit-comparison task, errors were not different across the two trials in which NP was assessed (related $v s$ unrelated) for the middleaged participants, $\mathrm{T}=3.00, \mathrm{Z}=1.57, \mathrm{P}=$ 0.116 , or older participants, $\mathrm{T}=8.50, \mathrm{Z}=$ $0.93, \mathrm{P}=0.353$. This result makes it unlikely that slowing for the related condition was compromised by speed/accuracy trade-off. Older adults made significantly more errors when the target was in a digit-pair than when the target was presented with a non-conflicting stimulus (unrelated $v s$ asterisk), $\mathrm{T}=0.00$, $\mathrm{Z}=2.20, \mathrm{P}<0.028$. This finding followed the same pattern as that observed for the naming latencies, with slowing when a comparison between conflicting stimuli was required. Overall errors were not significantly different between groups, i.e., for related trials, $\mathrm{U}=17.50, \mathrm{Z}=-1.52, \mathrm{P}=0.127$; for asterisk trials, $\mathrm{U}=32.00, \mathrm{Z}=0.00, \mathrm{P}=1.00$; and for unrelated trials, $U=17.50, Z=-1.68$, $\mathrm{P}=0.093$, although older adults tended to make more errors than middle-aged adults in trials with unrelated digit-pairs. Thus, no age differences existed for the error rates in the related condition. Therefore, the speed/accuracy trade-off differences between age groups did not limit the strength of the finding that NP was absent for the group of older adults.

For the location-based task, the critical conditions were related and unrelated targetplus-distractor trials for measuring NP, and target-alone and unrelated trials for measuring the interference by the distractor. Again, older adults were significantly slower than middle-aged adults, $\mathrm{F}(1,14)=6.21, \mathrm{P}<$ 0.026 . The main effect of conditions was also significant, $\mathrm{F}(2,28)=13.98, \mathrm{P}<0.0001$, with significantly slower responses in related trials, which presented the target at a recently ignored location, and faster responses in target-alone trials. The interaction between age and condition was not significant, $\mathrm{F}(2$, 28) $=0.28, P=0.759$.

The absolute differences in median response time for distractor interference (unrelated minus target alone) and locationbased NP (related minus unrelated) were submitted to separate ANOVA, with age as a random between-subject factor. There was no difference between the distractor interference scores for the older participants' performance and those observed for the middle-aged group, $\mathrm{F}(1,14)=0.24, \mathrm{P}=$ 0.629 . As expected from previous findings in the literature, the magnitude of spatial NP did not differ significantly between the older and the middle-aged groups, $\mathrm{F}(1,14)=0.14, \mathrm{P}=$ 0.714 . Similar analyses conducted on proportional effects of distractor (interference $=$ (unrelated - target alone)/unrelated and NP = (related - unrelated)/unrelated) yielded a similar pattern of significant results.

Error data analyses showed that neither differences between related and unrelated trials for the middle-aged, $\mathrm{T}=1.50, \mathrm{Z}=1.27$, $\mathrm{P}=0.201$, and older participants, $\mathrm{T}=16.00$, $\mathrm{Z}=0.28, \mathrm{P}=0.779$, nor differences between unrelated and target-alone trials for the middleaged, $\mathrm{T}=0.00, \mathrm{Z}=1.83, \mathrm{P}=0.068$, and older participants, $\mathrm{T}=8.00, \mathrm{Z}=0.73, \mathrm{P}=0.463$, 
were significant. Older adults made more errors under interference from the distractor in unrelated trials, $\mathrm{U}=12.00, \mathrm{Z}=-2.10, \mathrm{P}=$ 0.036 , but there were no different error rates across age groups in related, $\mathrm{U}=24.00, \mathrm{Z}=$ $-0.84, \mathrm{P}=0.401$, and target-alone trials, $\mathrm{U}=$ $24.00, \mathrm{Z}=-0.84, \mathrm{P}=0.401$.

\section{Comparisons of negative priming measures across tasks}

Finally, proportional NP scores (displayed in Figure 3) for each task, i.e., the paper-andpencil digit-comparison task, the computerized digit-comparison task, and the localbased task, were assessed by 2 (age: middleage $v s$ older) x 3 (task) MANOVA, with age as a random effect. The age effect was significant, Rao R $(3,12)=16.02, \mathrm{P}<$ 0.0002. Post hoc comparisons revealed that the performance of older adults showed diminished NP for both paper-and-pencil ( $\mathrm{P}$ $<0.027)$ and computerized digit-comparison tasks $(\mathrm{P}<0.0007)$ when compared to the magnitude of NP for the middle-aged participants, whereas both older and middle-aged groups showed spatial NP to an equivalent extent $(\mathrm{P}=0.728)$. MANOVA conducted on the absolute differences between the critical conditions, related $v s$ unrelated, yielded a significant effect of age, Rao R $(3,12)=$ $14.90, \mathrm{P}=0.0002$, with a significant reduction of NP in the older group performance in the computer-implemented digit-comparison task $(\mathrm{P}<0.0006)$, approached significance in the paper-and-pencil digit-comparison task $(\mathrm{P}=0.070)$, and absence of significant difference in spatial NP $(\mathrm{P}=0.714)$.

The primary results from this experiment show not only that NP is reliable for the paper-and-pencil digit-comparison task but also a dissociation between the kind of NP derived from digit-comparison tasks and the NP from the spatial task which appears to be evident from older adults' performance. This finding is consistent with earlier reports showing that older adults, unlike younger adults, appear to be unable to suppress the identity of irrelevant stimuli while they show reliable suppression of location (12). For older adults, the magnitude of proportional NP from the location-based task, but not from the digitcomparison tasks, was equivalent to the NP for middle-aged adults, thus providing evidence that the paper-and-pencil digit-comparison task may measure another type of NP that is different from the spatial one.

\section{Discussion}

The central issue examined in this investigation was whether reliable slowing in responses associated with ignored repeated targets, i.e., NP, can be detected and measured by means of a new and practical paperand-pencil version of a digit-comparison task. A secondary aim of this research was to explore the magnitude of this effect of NP in older adults, for whom failures to demonstrate NP may be particularly evident in some selective attention tasks that require identification of a stimulus which had been previously disregarded as being a distractor. The magnitude of NP from the paper-and-pencil task was assessed in younger, middle-aged and older adults, and age-related differences in types of NP were studied across digitcomparison and location-based tasks.

The paper-and-pencil digit-comparison task appears to be simple, it can be easily and rapidly administered, and detects NP, as indicated by slowing to complete the condition with related items (with the distractor in a digit-pair appearing as a target in the next digit-pair) relative to the condition with unrelated digit pairs. Three separate marking times are recorded, one for each list condition (i.e., one list containing non-conflicting pairs, a second list with unrelated digit-pairs, and a third list with related digit-pairs), which permit a baseline measure of performance to estimate the additional time needed to make the choice of the target by comparisons between digits and provide an index of NP of 
slowing for recently unselected targets. Using proportional scoring of differences across lists may be pertinent here because overall older adults perform more slowly in the tasks, as it became evident in Experiment 3, and because of previous models of performance in selective attention tasks that suggest a uniform increment in latency in normal aging rather than a phenomenon requiring process-specific explanations (33). While the age groups exhibited a different baseline in marking times, manipulating the complexity of target selection had no demonstrable differential influence on proportional (or absolute differences) measures of performance across the age groups. On the basis of the assumption that the variation of older individuals' performance obeyed a general slowing instead of depending on the type of information processes involved, proportional indices can provide an appropriate way to interpret possible age differences in performance in the related condition.

Data analyses clearly showed a reliable effect of NP; i.e., subjects were slower in completing the form containing the related digit-pairs than in completing the list with unrelated digit-pairs. In the last version of the task, performance in the unrelated condition proved to be faster, before or after the related condition. This finding is inconsistent with the claim that slowing is produced by expectancy biases developed across the lists. NP appears to be present to the same extent for younger and middle-aged adults, in contrast to the performance of older adults for whom NP was reliably diminished. That is, older adults did not seem to complete the related list more slowly, suggesting that the paperand-pencil digit-comparison task may tap a kind of NP that appears to be impaired by aging, possibly reflecting the existence of age-related deficits in distractor suppression such as those proposed in previous developmental studies $(6,15)$.

According to this theory, a reduction in the efficiency of inhibitory mechanisms may underlie age differences in a variety of cognitive tasks so that the capacity of controlling access to and sustaining the non-relevant information in working memory may be impaired in aging under some circumstances, explaining the failure to gate out of working memory stimuli and thoughts that are not directly relevant to current goals. However, multiple inhibitory systems have been proposed, not all of which are reduced in effectiveness with age. While in identity suppression tasks (i.e., where the distractor in a pair reappears as the target on the next pair) older adults typically show reduced or no NP compared to younger adults, studies employing specific location-based tasks (i.e., where target in a current display appears in the same location as the distractor in the preceding display) have demonstrated that older adults show as much NP as younger adults $(12,14)$. Indeed, in testing a group by priming interaction, it is necessary to proceed with caution to affirm the overall absence of NP in normal aging, since there exist several reports of reliable NP in older adults compared to younger adults not only in spatial tasks but also in identity tasks $(10,27$, 31,34). Besides the type of NP involved, mnemonic strategies which could be elicited to some extent by experimental contextual variables (e.g., difficulty in perceptual selection, reduction of the probe display exposure) seem to affect the age group by priming interaction $(13,39)$, with evident NP for older adults only demonstrable under conditions designed to encourage episodic retrieval. However, what processes moderate negative priming in aging is still under debate $(27,40)$.

Although this issue is not resolved here, the results of the present study (Experiment 3 ) are consistent with the previous statement that NP derived from tasks that require suppressing the identity of distractors would be less robust in older adults. Sparing of spatial NP in the older group is consistent with the hypothesis that inhibitory mechanisms that 
operate to prevent processing of irrelevant locations seem to be preserved in aging.

The paper-and-pencil digit-comparison task uses the referent-size selection procedure, a variant of the NP procedure that ensures that both targets and distractors are attended through comparisons between their semantic referents, instead of using a typical NP procedure requiring participants to attend a feature of the target while ignoring the distractor. The referent-size selection procedure is not exclusive to the present study. It has been explored recently $(5,27)$ by using computerized tasks and words as stimuli (e.g., participants were asked to read the name of the larger animal, or the heavier noun), with the finding that NP not only occurred when the to-be-ignored distractor was overtly attended but was also actually enhanced in this procedure. This report has theoretical and practical implications. It goes against the view that NP is presumably a measure of pure attentional inhibition arising from ignoring prime distractors or not directing attention to them, by which deep processing of distractors as that involved in decision-type tasks that afford post-lexical processing should prevent NP. On this basis, participants that were able to manifest awareness upon NP manipulation were excluded from further data analysis in several studies (e.g., 3,12). The finding of reliable NP for attended distractors is not consistent with the notion that a mechanism that limits phenomenal awareness to relevant items only underlies NP (5). This issue is also important for appropriate interpretations when NP tasks are used in clinical groups. A practical implication relates to the increased magnitude of NP when the procedure involves overtly attending distractors relative to the typical NP procedures, by which the referent-size selection procedure seems to be more suitable for the clinical-experimental setting.

Diminished NP in older adults in tasks with an overtly attended distractor may support a memory model, because this type of task favors greater depth of processing in which episodic retrieval would be advantageous. On the basis of the assumption that the semantic representation of both attended target and distractor is positively primed, response times to these items in subsequent trials may be facilitated. Further faster response time to the attended distractor at its occurrence, however, adds to slowing of the response derived from a "do not respond to" tag that became associatively linked with its semantic memory representation, which is automatically retrieved when a similar object is subsequently encountered. These encoding and retrieval mechanisms that mediate NP have been proposed to make distracting information less available to flow automatically into actions and enable coherent goaldirected behavior, and they may vary with age. NP seems to reflect cognitive processes that are functionally important for effective attentional processing and executive functioning and that could be partially compromised by age-related factors.

Intense efforts are currently being made in an attempt to obtain a clearer picture of executive functioning by carrying out experimental tests of cognitive models. Executive processes are thought of in terms of a general attentional resource involved in several dayto-day abilities such as reasoning, decision making, comprehension, and memory (1). As explained above, NP has been broadly investigated because of its relevance to theories of selective attention. That is, NP is typically said to measure either attentional inhibition or episodic memory retrieval. There exist many kinds of NP tasks which have been developed to understand NP, an effect that constitutes a challenge for the most current theories of attention. More sophisticated neuropsychological tests could be valuable for advances in specifying more precisely how different NP tasks and conditions can moderate the processes involved in NP, which vary in both direction and magnitude. On the other hand, a promising application to 
the diagnosis of cognitive dysfunction requires the development of simple tasks that are suitable for neuropsychological clinical routines. Computerized NP tasks broadly used in the studies reviewed often present several problems for day-to-day clinical practice. They require expensive and specialized equipment and a more controlled environment (e.g., technical parameters of equipment and software, luminance of the display and testing room) such that standardization becomes a difficult goal. Reading-based NP tasks show constraints under some circumstances, such as when the population presents heterogeneity in the level of reading ability.

Since the paper-and-pencil digit-comparison task can be practically administered, it can be very useful for neuropsychologists seeking practical measures of NP that do not require cumbersome technical equipment within the clinical-experimental setting, involving within- and between-subject designs, since it has proved to be a sensitive and practical screening tool to index NP and to detect individual differences. In the present study, the number of errors and omissions were relatively low, and the method of administration and time recording were rapid and easy. The magnitude of NP from the digit-comparison task (with a mean differ- ence between the related and unrelated lists of approximately $6 \mathrm{~s}$ and a proportional NP of approximately $12 \%$ for younger and middleage adults, as can be seen in Table 2 and Figure 3) was considerably enhanced as compared with other non-computerized NP tasks with ignored distractors, e.g., with a mean difference of less than $1 \mathrm{~s}$ (proportional $\mathrm{NP}=4.57 \%$ ) in a previous study using the list procedure (30). In addition, sex or years of education did not appear to affect the NP score for the paper-and-pencil digit-comparison task. These characteristics of the task are favorable for the clinical setting and support further studies to determine and improve its effective utility as a neuropsychological tool.

\section{Acknowledgments}

I am indebted to Cesar Galera for guidance during all my Ph.D. studies and for providing the facilities for this research. Special thanks are due to Rolando P. Sylwan for advice and assistance during all stages of this research. I am also grateful to the anonymous reviewers of an earlier version of this article, and to Paul Stephaneck, J. Lino O. Bueno, Luiz Gawryszewski, and Luis Ribeiro do Valle for valuable comments that have benefited this work.

\section{References}

1. Baddeley AD, Della Sala S, Gray C, Papagno C \& Spinnler H (1997). Testing central executive functioning with a pencil-and-paper test. In: Rabbitt P (Editor), Methodology of Frontal and Executive Functions. Psychology Press, Hove, East Sussex, UK.

2. Dalrymple-Alford EC \& Budayr B (1966). Examination of some aspects of the Stroop colour-word test. Perceptual and Motor Skills, 23: $1211-1214$

3. Tipper SP (1985). The negative priming effect: inhibitory priming by ignored objects. Quarterly Journal of Experimental Psychology, 37A: 571-590.

4. Fox $E$ (1995). Negative priming from ignored distractors in visual selection: a review. Psychonomic Bulletin and Review, 2: 145-173.

5. MacDonald PA \& Joordens S (1999). Negative priming effects that are bigger than a breadbox: attention to distractors does not eliminate negative priming, it enhances it. Memory and Cognition, 27: 197-207.

6. Tipper SP (1991). Less attentional selectivity as a result of declining inhibition in older adults. Bulletin of the Psychonomic Society, 29: 45-47.

7. Beech A, Powell T, McWilliam J \& Claridge G (1989). Evidence of reduced "cognitive inhibition" in schizophrenia. British Journal of Clinical Psychology, 28: 109-116.

8. Stuss DT, Toth JP, Franchi D, Alexander MP, Tipper S \& Craik FIM (1999). Dissociation of attentional processes in patients with focal frontal and posterior lesions. Neuropsychologia, 37: 1005-1027.

9. Metzler C \& Parkin AJ (2000). Reversed negative priming following frontal lobe lesions. Neuropsychologia, 38: 363-379.

10. Sullivan MP, Faust ME \& Balota D (1995). Identity negative priming in older adults and individuals with dementia of the Alzheimer type. Neuropsychologia, 9: 537-555.

11. Stout JC, Wylie SA, Simone PM \& Siemers ER (2001). Influence of competing distractors on response in Huntington's disease and Parkinson's disease. Cognitive Neuropsychology, 18: 643-653.

12. Connelly SL \& Hasher L (1993). Aging and the inhibition of spatial 
location. Journal of Experimental Psychology: Human Perception and Performance, 19: 1238-1250.

13. Kane MJ, May CP, Hasher L, Rahhal T \& Stoltzfus ER (1997). Dual mechanisms of negative priming. Journal of Experimental Psychology: Human Perception and Performance, 23: 632-650.

14. Simone PM \& McCormick EB (1999). Effect of a defining feature on negative priming across the life span. Visual Cognition, 6: 587-606.

15. McDowd JM \& Oseas Kreger DM (1991). Aging, inhibitory processes, and negative priming. Journal of Gerontology, 46: 340345.

16. Houghton G \& Tipper SP (1994). A model of inhibitory mechanisms in selective attention. In: Dagenbach D \& Carr TH (Editors), Inhibitory Processes in Attention, Memory, and Language. Academic Press, New York.

17. Milliken B, Joordens S, Merikle PM \& Seiffert AE (1998). Selective attention: a reevaluation of the implications of negative priming. Psychological Review, 105: 203-229.

18. Neill WT, Valdes LA, Terry KM \& Gorfein DS (1992). Persistence of negative priming: II. Evidence for episodic trace retrieval. Journal of Experimental Psychology: Learning, Memory, and Cognition, 18: 993-1000.

19. May CP, Kane MJ \& Hasher L (1995). Determinants of negative priming. Psychological Bulletin, 118: 35-54.

20. Tipper SP (2001). Does negative priming reflect inhibitory mechanisms? A review and integration of conflicting views. Quarterly Journal of Experimental Psychology. Section A, 54: 321-343.

21. Tipper SP, MacQueen GM \& Brehaut JC (1988). Negative priming between response modalities: evidence for the central locus of inhibition in selective attention. Perception and Psychophysics, 43: 45-52.

22. Tipper SP, Eissenberg T \& Weaver B (1992). The effects of practice on mechanisms of attention. Bulletin of the Psychonomic Society, 30: 77-80.

23. Neill WT (1977). Inhibitory and facilitatory processes in selective attention. Journal of Experimental Psychology: Human Perception and Performance, 3: 444-450.

24. Tipper SP, Weaver B, Cameron S, Brehaut JC \& Bastedo J (1991). Inhibitory mechanisms of attention in identification and localization tasks: time course and disruption. Journal of Experimental Psychology: Learning, Memory, and Cognition, 17: 681-692.

25. Neill WT \& Valdes LA (1992). Persistence of negative priming: steady state or decay? Journal of Experimental Psychology: Learning, Memory, and Cognition, 18: 565-576.

26. Tipper SP, Weaver B \& Milliken B (1995). Spatial negative priming without mismatching: comment on Park and Kanwisher (1994). Journal of Experimental Psychology: Human Perception and Per- formance, 21: 1220-1229

27. Pesta BJ \& Sanders RE (2000). Aging and negative priming: is ignored information inhibited or remembered? Experimental Aging Research, 26: 37-56.

28. Goldman Rakic PS (1995). Architecture of the prefrontal cortex and the central executive. Annals of the New York Academy of Sciences, 769: 71-83.

29. Neill WT \& Westberry RL (1987). Selective attention and the suppression of cognitive noise. Journal of Experimental Psychology: Learning, Memory, and Cognition, 13: 327-334.

30. Tipper SP \& Cranston M (1985). Selective attention and priming: inhibitory and facilitatory effects of ignored primes. Quarterly Journal of Experimental Psychology, 37A: 591-611.

31. Langley LK, Overmier JB, Knopman DS \& Prodhomme MM (1998). Inhibition and habituation: Preserved mechanisms of attentional selection in aging and Alzheimer's disease. Neuropsychology, 12: 353-366.

32. Bestgen $Y$ \& Dupont $V$ (2000). Is negative priming a reliable measure for studying individual differences in inhibition? Current Psychology of Cognition, Cahiers de Psychologie Cognitive, 19: 287-305.

33. Cerella J (1991). Age effects may be global, not local: Comment on Fisk and Rogers. Journal of Experimental Psychology: General, 120: 215-233.

34. Verhaegen P \& De Meersman L (1998). Aging and the negative priming effect: a meta-analysis. Psychology and Aging, 13: 435444.

35. Folstein MF, Folstein SE \& McHugh PR (1975). "Mini-mental State". A practical method for grading the cognitive state of patients for the clinician. Journal of Psychiatric Research, 12: 189198.

36. May CP \& Hasher L (1998). Synchrony effects in inhibitory control over thought and action. Journal of Experimental Psychology: Human Perception and Performance, 24: 363-379.

37. Horne JA \& Ostberg O (1976). A self-assessment questionnaire to determine morningness-eveningness in human circadian rhythms. International Journal of Chronobiology, 4: 297-310.

38. Schneider W (1988). Micro experimental laboratory: an integrated system for IBM-compatibles. Behavior Research Methods, Instruments, and Computers, 20: 206-217.

39. Gamboz N, Russo R \& Fox E (2000). Target selection difficulty, negative priming, and aging. Psychology and Aging, 15: 542-550.

40. Grant JD \& Dagenbach D (2000). Further considerations regarding inhibitory processes, working memory, and cognitive aging. American Journal of Psychology, 113: 69-94. 